\title{
EFEITO CRÔNICO DA SACAROSE E DO ALOPURINOL NO COMPORTAMENTO DIETÉTICO E NO TECIDO ADIPOSO BRANCO EM RATOS WISTAR
}

\author{
Bárbara Gatti Cardoso*, William Gustavo de Lima, Maíra de Castro \\ Lima, Valéria Ernestânia Chaves
}

Universidade Federal de São João Del-Rei, Divinópolis/MG

*e-mail: gatti.ufsj@gmail.com

\section{Resumo}

Estudos apontam que o consumo excessivo de sacarose, como fonte de frutose, esta ligado a quadros de hiperuricemia, o qual tem papel chave na fisiopatologia da síndrome metabólica. Neste contexto se destaca a utilização do alopurinol, um fármaco hipouricêmico, porém evidências da sua utilização na síndrome metabólica ainda são escassas, o que motiva avaliações mais profundas. Este trabalho busca analisar a participação do alopurinol e da sacarose na dieta e no tecido adiposo branco retroperitonial (TAB retro) e epididimal (TAB epi) em ratos. Ratos wistar machos foram avaliados por um período de 16 semanas, sendo divididos em 4 grupos: $\mathbf{C}$ : dieta controle $(n=5)$; $\mathbf{S}$ : dieta controle e água com 30\% em sacarose ad libitum ( $\mathrm{n}=5)$; $\mathbf{S A}$ : dieta do grupo $\mathrm{S}$ e alopurinol administrado por gavagem $(30 \mathrm{mg} / \mathrm{kg} / \mathrm{dia})(\mathrm{n}=6)$, CA: dieta do grupo $\mathrm{C}$ e alopurinol administrado por gavagem $(\mathrm{n}=6) \quad(30 \mathrm{mg} / \mathrm{kg} / \mathrm{dia})$. Decorrido o experimento, os animais foram eutanasiados e o TAB retro e TAB epi foram coletados e pesados, sendo os resultados avaliados pelo Two Way ANOVA com $\mathrm{P}<0,05$. A ingestão alimentar dos grupos $\mathrm{C}$ e $\mathrm{CA}$ foi significativamente maior que nos grupos $\mathrm{S}$ e SA, e a ingestão hídrica obedeceu a esta tendência nas quatro primeiras semanas, a partir da qual os valores não se diferiram estatisticamente. O ganho de peso foi semelhante entre os grupos. O TAB epi resultou em 6,23g; $6,94 \mathrm{~g} ; 5,85 \mathrm{~g}$ e $9,00 \mathrm{~g}$ nos grupos $\mathrm{C}, \mathrm{S}$, CA e SA respectivamente, sem diferença estatística entre eles. O TAB retro apresentou valores de 3,56g; 6,96g; 3,99g e $7,61 \mathrm{~g}$ nos grupos $\mathrm{C}, \mathrm{S}, \mathrm{CA}$ e $\mathrm{SA}$ respectivamente, possuindo diferença estatística entre os grupos S e C (aumento de 95,5\%), SA e C (aumento de $113,8 \%$ ), SA e CA (aumento de 90,7\%), e S e CA (aumento de 74,44\%). Estes 
resultados mostram que o alopurinol tem pouca influencia sobre o consumo hídrico e alimentar, e que não reverte o aumento de TAB induzido pela sacarose, sendo seu uso crônico adjuvante deste processo.

Palavras-chave: Síndrome metabólica, Alopurinol, Sacarose. 